\title{
Atom Probe Tomography of III-Nitrides Based Semiconducting Devices
}

\author{
Ravi Shivaraman ${ }^{1}$, Yuh-Renn $\mathrm{Wu}^{2}$, Soojeong Choi ${ }^{1}$, Roy Chung ${ }^{1}$ and James Speck ${ }^{1}$ \\ ${ }^{1}$ Materials Department, University of California, Santa Barbara, United States \\ ${ }^{2}$ Department of Electrical Engineering Department, National Taiwan University, Taipei, Taiwan
}

Commercial Light emitting devices are grown on c-plane Gallium Nitride. The active region - where the photons are generated - is an Indium Gallium Nitride quantum well (QW). The radiative recombination efficiency of these light emitters is related to the carrier charge density distribution and transport across and within this active region. The charge density and charge transport in the device is governed by the potential landscape that charges move through. This potential landscape is intimately related to the Indium distribution in inside the quantum well. For instance, higher indium regions experience larger strain and hence greater piezoelectric field contribution and also a greater electrostatic potential, leading to deeper troughs in the energy landscape. Atom probe tomography (APT) emerges as an ideal characterization tool to map this 3-D Indium distribution. APT combines time-of-flight measurement with point projection imaging [1]. The APT analysis of the c-plane GaN/InGaN LED was performed using the Cameca Local Electrode Atom Probe 3000XHR. The epi-structure of the LED for APT analysis was obtained from Walsin Lihwa Corp. It consisted of 9 pairs of QW/quantum barrier (QB) structures, where the barrier thickness was around $10 \mathrm{~nm}$ and well thickness around $2.5 \mathrm{~nm}$. The reconstructed APT data which delineates the LED heterostructure is shown in Figure 1(a). The average quantum well III-site Indium concentration was estimated to be $\sim 16 \%$. The barrier/quantum well interfaces are not abrupt as can be seen in Figure 1(b). There is an asymmetry in the chemical diffusivity at the upper and lower interfaces - the upper interface being more chemically diffuse. A difference plot between experimentally observed and simulated binomial bin distributions reveals that the Indium distribution is random (Figure 1(c)).

These indium composition maps obtained from APT experiments were employed to generate the potential landscape in device simulations. Recently, in their work on 2-D LED device modeling, Wu et. al. [2] assumed an Indium distribution where the lateral variations were assumed sinusoidal and the composition variations in the growth direction were characterized by a Gaussian. Their result (Figure 1(d)) showed that by considering the indium composition fluctuations in the QW, the low forward voltage in the commercial blue LED diode can be explained. The influence of indium fluctuation to the IQE and carrier transport is very significant, and thus cannot be ignored in the device design and modeling.

Another technologically promising ternary III-nitride material system is InAIN - a promising candidate for both optoelectronic and electronic devices. InAlN with $18 \%$ In concentration has comparable a-axis lattice constant to $\mathrm{GaN}$, which makes it possible to grow lattice-matched (0001) structures. In the work by Choi et. al. [3], InAIN layers were grown on commercial (0001) Ga-face Lumilog GaN templates by plasma assisted molecular beam epitaxy. The InAlN layer was grown on a GaN-buffer at $480^{\circ} \mathrm{C}$ under N-rich conditions. The fluctuations of Indium concentration was observed quantitatively in APT. Figure 
2(a) shows the reconstructed 3D image of Indium distribution. Indium segregated regions, propagating in the [0001] direction, are clearly observed. The microstructure is comprised of columnar cells of $\sim 10$ $\mathrm{nm}$ lateral length scale. The Indium III site mole fraction, at the cell center is less than 0.03 , while it is over 0.26 in the cell boundary (see inset 2(c)). The average Indium mole fraction measured to be 0.22. A similar study that employed metal organic chemical vapor deposition (MOCVD) to grow latticematched InAlN layer on Ga-face GaN/Sapphire (0001) was carried out by Chung et. al. [4]. Atom probe analysis of the InAIN layer revealed a uniform distribution of Indium (Figure 2(b)). A difference plot between experimentally observed and simulated binomial bin distributions indicated that the Indium distribution is random (Figure 2(d)). Thus MOCVD grown InAIN morphology contrasts with that obtained via PAMBE - and both InAIN morphologies seem to hold potential for new applications.

Atom Probe Tomography has thus shown great promise in characterization of III-nitrides and enabled to extract quantitative metrics on elemental distribution in the III-nitride heterostructures.

\section{References:}

[1] M. K. Miller in “Atom Probe Tomography: Analysis at the Atomic Level”, (Kluwer Academic/Plenum Publishers, New York) p.121

[2] Y-R. Wu, R. Shivaraman., K-C. Wang and J.S. Speck, Appl. Phys. Lett., 101, 083505 (2012)

[3] S-J. Choi, F. Wu, R. Shivaraman., E.C. Young and J.S. Speck, Appl. Phys. Lett. 100, 232102 (2012)

[4] R. B. Chung, F. Wu, R. Shivaraman., S. Keller, S. P. DenBaars, J. S. Speck and S. Nakamura, Journal of Crystal Growth, 324, 1 (2011) p.163

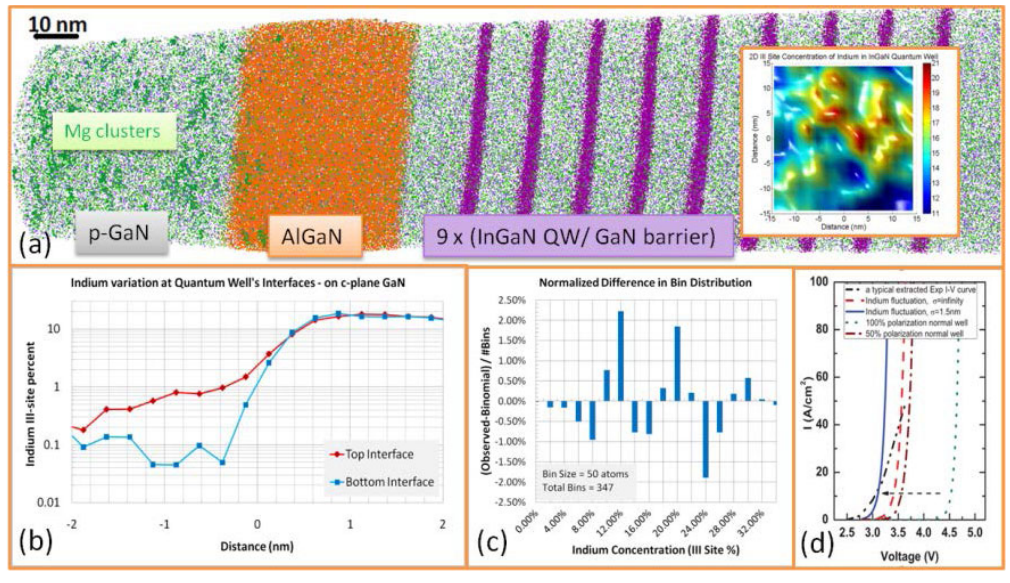

Figure 1 (a) Reconstructed APT data of c-plane InGaN/GaN LED device (inset) - 2-D composition map of Indium in the plane of a Quantum well, (b) Compositional variation of Indium at upper \& lower interfaces of a Quantum well, (c) Difference plot between Observed \& Binomial bin distributions, (d) Simulated I-V curves from ref. [2] compared to experiment

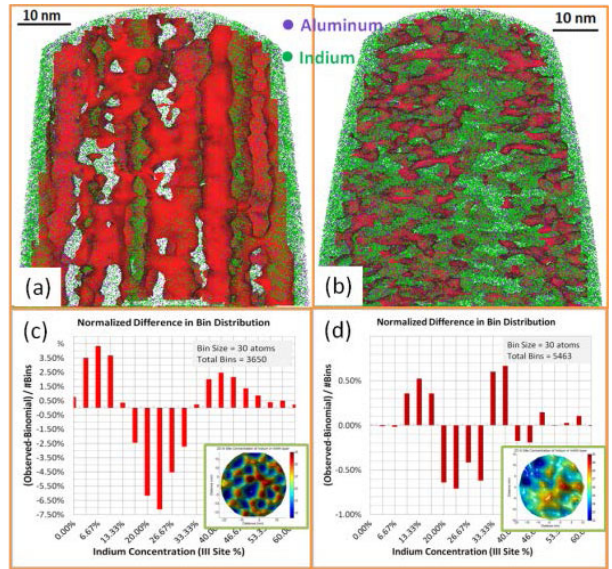

Figure 2 (a), (b) In and $\mathrm{Al}$ atom probe tomography maps in an InAlN layer. Iso-concentration surfaces (red) are constructed at 24 III-site \% Indium. (c), (d) Difference plot between Observed \& Binomial bin distributions ((c), (d) inset - Indium III-site distribution in plane perpendicular to the growth direction) 